\title{
ANÁLISIS DE LA RELACIÓN ENTRE LAS ALTAS CAPACIDADES INTELECTUALES Y LA INTELIGENCIA EMOCIONAL EN NIÑOS Y NIÑAS DE 8 A 10 AÑOS DE LA CIUDAD DE CÓRDOBA, ARGENTINA
}

\author{
ANALYSIS OF THE RELATIONSHIP BETWEEN THE HIGH \\ INTELLECTUAL CAPACITIES AND THE EMOTIONAL INTELLIGENCE \\ IN BOYS AND GIRLS FROM 8 TO 10 YEARS OLD OF THE CITY OF \\ CÓRDOBA, ARGENTINA
}

\author{
Paula Irueste ${ }^{2}$ \\ Camila Lilén Arsaut ${ }^{3}$ \\ María Agustina Benavidez Ferre ${ }^{4}$
}

\section{RESUMEN}

El presente estudio se centró en analizar la posible relación entre las altas capacidades intelectuales (ACI) y la inteligencia emocional (IE) en niños y niñas de 8 a 10 años de la Ciudad de Córdoba, Argentina. Se llevó a cabo un estudio cuantitativo, explicativo, transversal y correlacional mediante la aplicación de dos instrumentos-pruebas estandarizados: BADyG E2-r de Yuste (2014) y EQ-i:YV de Bar-On (1997). Estos fueron aplicados a 99 participantes. A partir de los resultados obtenidos no se identificaron diferencias sustanciales en relación a las puntuaciones de IE entre los niños y niñas que presentaron indicadores $\mathbf{A C I}(42,5 \%)$ (Talentos simples y talentos múltiples), en relación al grupo control (46,5\%). En general, los alumnos/as con indicadores $\mathrm{ACl}$ del presente estudio autoinformaron un buen ajuste emocional, buena capacidad de adaptabilidad y habilidades intra e interpersonales, así como adecuadas estrategias para manejar el estrés.

Palabras claves: altas capacidades Intelectuales - inteligencia emocional - talento

\section{ABSTRACT}

The present study focused on analyzing the possible relationship between high intellectual abilities (ICA) and emotional intelligence (EI) in children aged 8 to 10 years old in the City of Córdoba, Argentina. A quantitative, explanatory, transversal and correlational study was carried out through the application of two standardized test instruments: BADyG E2-r from Yuste (2014) and EQ-i: YV from Bar-On (1997). These were applied to 99 participants. Based on the results obtained, no significant differences were identified in relation to the IE scores among the children who presented ACl indicators (42.5\%) (Simple talents and multiple talents), in relation to the control group (46.5\%) In general, the students with $\mathrm{ACl}$ indicators of the present

2 paulairueste@gmail.com

3 camii.arsaut@gmail.com

4 agustinabenavidezferre@gmail.com 
study self-reported good emotional adjustment, good adaptability and intra and interpersonal skills, as well as adequate strategies to manage stress.

Keywords: high intellectual abilities - emotional intelligence - talent.

\section{INTRODUCCIÓN}

Hoy en día, en el contexto que nos atraviesa, las altas capacidades intelectuales (ACl) nos brindan la posibilidad de mirar a los niños y niñas desde una nueva perspectiva más integral. Creemos necesario dejar de mirar desde una visión de pérdida o falta, para observar desde la potencialidad (aquello que los niños y niñas son capaces de hacer). Conjuntamente que se fue fortaleciendo el término $\mathrm{ACl}$, se generaron nuevas preguntas al respecto del área emocional de estos niños y niñas. Es así que, para intentar responder a esos enigmas, el presente estudio se impulsó a investigar acerca de las posibles relaciones entre las altas capacidades intelectuales y la inteligencia emocional en niños y niñas de 8 a 10 años de la Ciudad de Córdoba, Argentina.

El estudio de la superdotación y el talento en el ámbito de la Psicología cuenta con antecedentes sólidos desde hace décadas. Sin embargo, no sucedió lo mismo en Argentina, donde actualmente se llevan a cabo grandes esfuerzos para dar respuesta a demandas que se presentan tanto en el ámbito familiar como en el educativo. Si bien la ley de Educación menciona la atención a alumnos y alumnas con altas capacidades intelectuales no se llevan a cabo respuestas educativas específicas y diferenciales.

Tanto las familias como las escuelas, necesitan información, asesoramiento y soporte para que el proceso educativo de la población infantil con altas capacidades intelectuales (ACI) sea exitoso, entendiendo que ese éxito se consigue cuando pueden formarse los canales de comunicación y colaboración adecuados entre estos sistemas significativos para la infancia.

Por otro lado, el estudio de la Inteligencia Emocional resulta aún más desconocido en los avances científicos de este país. Desde los inicios, de la mano de Gardner (1993) al ampliar el paradigma de conocimiento con su Teoría de las Inteligencias Múltiples, como el puntapié inicial de Goleman (1995) adentrándose en la temática de la Inteligencia Emocional, hubo una evolución en su estudio que es necesario abordar.

\section{APROXIMACIÓN CONCEPTUAL}

\section{Altas capacidades intelectuales}

El concepto de Alta Capacidad Intelectual, engloba los conceptos de superdotación y talento. Sastre-Riba (2008) menciona que la alta capacidad consiste en una potencialidad intelectual elevada que es lo que la caracteriza, no un rasgo de personalidad, ni una conducta escolar o un rendimiento concreto. La superdotación y el talento (simple o múltiple), son las formas en que se expresa la Alta Capacidad Intelectual.

De este modo, para esclarecer el término de altas capacidades intelectuales, citaremos a Comes, Díaz, Luque \& Moliner (2008), quienes definen al individuo con Altas Capacidades Intelectuales como aquel alumno que reclama una atención diferenciada, ya que precisa de manera indispensable una respuesta educativa acorde a sus capacidades para que su inclusión en el ámbito escolar sea exitosa. Por otro lado, Manzano \& Arraz (2008) describen a los niños con altas capacidades intelectuales, como sujetos que obtienen rendimientos con las mismas características que los niños superdotados, pero que además tienen motivación y creatividad para llevar a cabo su tarea.

Castelló (2008) afirma que las altas capacidades implican un conjunto de características cognitivas que permiten llevar a cabo operaciones de representación y procesamiento de alto rendimiento y cuya determinación se fundamenta en la existencia de un conjunto estable de recursos intelectuales, el cual aporta los mecanismos para representar y procesar objetos de manera eficiente. A la vez, siguiendo a Ferrándiz (2011), los niños superdotados y con talento son sujetos caracterizados por un alto rendimiento debido a las aptitudes excepcionales que poseen. Son niños que requieren respuestas educativas diferentes de las que suele ofrecer el programa escolar ordinario, para que sea posible contribuir a su desarrollo y, por extensión, al de la sociedad. 


\section{Inteligencia emocional}

Los primeros precedentes de la IE los encontramos en un periodo en el que inteligencia y emoción eran constructos independientes y pertenecían a campos de estudio contrapuestos. Binet y Simon (1908, citado en Belmonte Lillo, 2013) estipularon dos tipos de inteligencia: una ideativa (similar a la de las teorías psicométricas clásicas) y otra instintiva (que operaba por medio de los sentimientos y se relaciona con la intuición). Aunque los autores no vincularon el proceso cognitivo y el emocional, apuntaron ya la idea de un uso inteligente de los sentimientos y de factores no puramente cognitivos.

Por su lado, Thorndike (1920, citado en Belmonte Lillo, 2013) se muestra como un predecesor claro e influyente de la IE cuando propone que los seres humanos poseen varios tipos de inteligencia, entre los cuales se encuentra la llamada inteligencia social. Ésta se define como la habilidad para entender a hombres y a mujeres, chicos y chicas, y para actuar sabiamente en las relaciones con las personas. Esta definición conlleva el uso de la empatía y la prosocialidad dentro de la inteligencia.

El constructo de IE se presentó formalmente definido en 1990 por Salovey y Mayer. Más tarde, la definición fue mutando hasta que en 1997 se formaliza la definición de la Inteligencia Emocional enfatizando más los aspectos cognitivos. Así se entiende la IE como:

La habilidad para percibir, valorar y expresar emociones con exactitud, la habilidad para acceder y/o generar sentimientos que faciliten el pensamiento; la habilidad para comprender emociones y el conocimiento emocional y la habilidad para regular las emociones promoviendo un crecimiento emocional e intelectual (Mayer \& Salovey, 1997, p.4).

La IE representa para los autores Salovey y Mayer, sobre todo, un grupo de habilidades alternativas a la inteligencia social. Por una parte, la IE resulta más amplia que la inteligencia social ya que incluye no sólo el razonamiento sobre las emociones en las relaciones sociales sino también el razonamiento sobre las emociones internas que son tan importantes para el crecimiento personal. Por otra parte, el nuevo constructo es más específico que la inteligencia social ya que principalmente atiende a los problemas emocionales encajados en los problemas personales y sociales.

MÉTODO

TIPO Y DISEÑO DE ESTUDIO: En esta investigación se llevó a cabo un Estudio Cuantitativo - Explicativo, transversal y correlacional mediante la aplicación de dos instrumentos estandarizados: Batería de aptitudes generales y diferenciales E2-r, de Yuste (2014) y el Inventario de Cociente emocional de Bar On (1997). Los mismos están situados dentro de la lógica objetivista y se caracterizan por presentar datos empíricos, producidos por los autores de la investigación.

PARTICIPANTES: Se llevó a cabo un muestreo no probabilístico de juicio, intencional o selección de expertos; tipo de muestreo que se caracteriza por ser el propio investigador quien elige a los sujetos que serán participantes de su estudio, basándose en las características que estos poseen. Dicha elección se realiza valorando la riqueza de los contenidos que estos actores pueden aportar a la investigación (Bologna, 2011).

La muestra estuvo constituida por 99 niños (44) y niñas (55), quienes estaban autorizados por sus madres y padres para participar de la investigación. En relación a las edades, el $43 \%$ presentó una edad de 8 años y el $56 \%$ de la muestra tenía 9 años.

Dichos niños y niñas asisten a instituciones educativas de la ciudad de Córdoba - Argentina, ya sea de modalidad pública, privada o semipública. Las instituciones educativas fueron seleccionadas a partir de una técnica de muestreo denominada homogénea, caracterizada por escoger a un sector de la población de acuerdo a ciertos criterios determinados intencionalmente por el investigador.

\section{INSTRUMENTOS}

Batería de Aptitudes Diferenciales y Generales (BADyG) E2. (Yuste Hernanz y Yuste Peña, 1980). 
La fecha de publicación del test original data en 1980, con renovación 2001, siendo que la fecha de la última revisión del test en su adaptación española. Baremos actualizados 2014.

El Consejo General de la Psicología (España), sostiene que el BADyG/E2-r (2001) es una batería de pruebas, de aplicación individual o colectiva, que permite evaluar seis aptitudes mentales diferenciales básicas y tres aptitudes complementarias, en escolares de $3^{\circ}$ y $4^{\circ}$ de educación primaria. A partir de las pruebas de aptitudes básicas se pueden obtener estimaciones de factores generales de segundo orden, concretamente de razonamiento lógico (RR), factor numérico (NN), factor verbal (VV) y factor visoespacial (EE), así como de un factor de inteligencia general (IG) de tercer orden. Por lo tanto, aborda la medición de la inteligencia desde un enfoque multidimensional jerárquico. Adicionalmente, a partir de la puntuación directa en IG, y teniendo en cuenta la edad cronológica del alumno, se puede obtener una puntuación de cociente intelectual (Cl). Finalmente, se ofrecen estimaciones de rapidez y eficacia. Es un test que ha sido muy utilizado, con revisiones y actualizaciones de los baremos

La fiabilidad de las puntuaciones se aborda desde la perspectiva de la consistencia interna (alfa de Cronbach) y de las dos mitades (coeficiente de Spearman-Brown y de dos mitades de Guttman). Los resultados de los análisis de fiabilidad son altos, ya que el alfa de Cronbach oscila entre .74 y .94, con valor promedio igual a .85. También es posible obtener intervalos de confianza en torno a las puntuaciones en inteligencia general (IG) y cociente intelectual (Cl).

Además, desde dicho Consejo, se considera de la facilidad de administración y corrección, otro de los puntos fuertes del BADyG/E2-r es que cuenta con baremos actualizados (2014) en muestras grandes de escolares españoles.

En Argentina, se ha realizado la validación del BADyG-i, en niños de 4 a 6 años. En la investigación se corrobora la fiabilidad del instrumento (0.905, en índice alfa de Cronbach) para medir inteligencia y obtener el perfil intelectual del estudiante de ese rango etario (Irueste \& Ferrándiz, 2016) lo que arrojó valores de consistencia interna óptimos para la validación de estas escalas.

Inventario de Cociente Emocional de Bar-On para jóvenes EQ-i:YV. (Emotional Quotient-Youth Version; Bar-On\& Parker, 2000)

El test está destinado a alumnos con edades comprendidas entre los 7 y los 18 años. El cuestionario consta de 60 afirmaciones en una escala tipo likert de 4 puntos ( 1 = nunca me pasa a $4=$ siempre me pasa). El objetivo de la escala es evaluar los componentes que describen la IE propuestos en su modelo (intrapersonal, interpersonal, adaptabilidad, manejo del estrés y estado de humor general).

Dichas escalas, están subdivididas en 15 subescalas que nombramos a continuación: 1) habilidad intrapersonal (autoconciencia emocional, asertividad, respeto personal, autoactualización, independencia); 2) habilidad interpersonal (relaciones interpersonales, responsabilidad social, empatía); 3) adaptabilidad (resolución de problemas, evaluación de la realidad, flexibilidad); 4) manejo del estrés (tolerancia al estrés, control de los impulsos); y 5) estado de ánimo general (alegría, optimismo). Además de evaluar estas 15 dimensiones el inventario ofrece cuatro indicadores de validez que miden el grado en el que los individuos responden al azar o distorsionan sus respuestas. Estos cuatro indicadores se crearon para reducir el efecto de deseabilidad social e incrementar la seguridad de los resultados obtenidos. Se entiende que el cuestionario proporciona información acerca de las competencias emocionales y sociales, permitiendo trazar un perfil social y afectivo (Bar-On, 1997; Bar-On \& Parker, 2000).

Entonces, el objetivo se centra en estudiar los componentes que definen a la Inteligencia Emocional y el procedimiento para evaluados. Dicho cuestionario fue adaptado al idioma español por medio de la labor del grupo de investigación de altas habilidades de la Universidad de Murcia (2011) y en población adolescente española, el instrumento ha sido validado por Ferrando (2006), en dicho estudio los coeficientes de consistencia interna de las escalas del instrumento oscilaron entre .69 y .83 .

\section{ANÁLISIS DE DATOS}

Para el análisis de datos cuantitativos se utilizó el paquete estadístico SPSS para Windows versión 20.0. Se realizó un análisis descriptivo, analizando medias, desviaciones típicas y distribución de las frecuencias. Se analizó la asociación entre ACl e IE en los niños y niñas que participaron del estudio mediante un análisis de Chi cuadrado. La estrategia metodológica que se adoptó responde a que, si bien ambas variables son con- 
tinuas, es a partir de un puntaje determinado que se considera que una persona cuenta con indicadores de ambas variables. No se optó por un análisis de correlación de Pearson, ya que esto no hubiera respondido a las características propias de los instrumentos utilizados ni a la pregunta de investigación. Para realizar esto se dividieron ambas variables de interés de acuerdo a los siguientes puntos de corte:

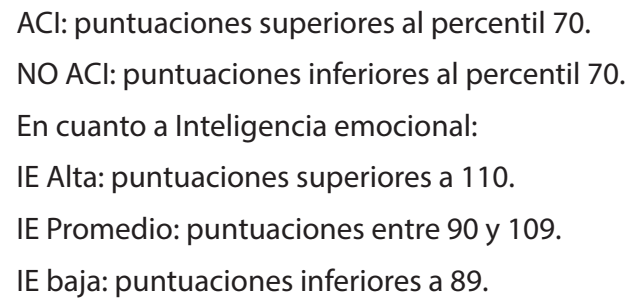

Así, se relacionaron las puntuaciones obtenidas de una variable con las puntuaciones obtenidas de la otra, con los mismos participantes o casos. (Hernández Sampieri, Fernández Collado y Baptista Lucio, 2006).

\section{CONSIDERACIONES ÉTICAS}

La investigación se llevó a cabo de acuerdo a los principios y reglas de acción contenidos en el Código de Ética de Fe.P.R.A. (2013), y aquellos establecidos en la Ley Nacional de Salud Mental, № 26.657 (2010). Principalmente, reconociendo a los participantes del estudio como sujetos portadores de derecho, haciendo hincapié en asegurar el derecho a la protección de la salud mental de todas las personas.

En este sentido, el Código de Ética de Fe.P.R.A. (2013) establece que resulta absolutamente esencial obtener el consentimiento informado de los sujetos de investigación o de sus representantes legales, con anterioridad a la administración del instrumento. Este principio pondera de manera fundamental la autonomía de la persona.

De igual modo, la Ley Nacional de Salud Mental (2010) resalta la importancia de solicitar un "consentimiento fehaciente" definido como la declaración de voluntad suficiente efectuada en este caso, por los representantes legales de los niños y niñas. Es necesario que sea emitido luego de recibir por parte del equipo, información clara, precisa y adecuada con el propósito de favorecer una comprensión racional de la magnitud de las decisiones tomadas. En este sentido, se explicitaron las condiciones y los alcances del procedimiento propuesto, los beneficios posibles de la investigación, la manera en la cual se efectuará la devolución, así como también se detallaron el número de contacto con las responsables del proyecto.

Durante la realización de este trabajo, la identidad de todos los consultantes (tanto adultos como niños/as) se mantuvo en total reserva. Esto último, corresponde al principio de respeto por los derechos y la dignidad de las personas que establece el Código de Ética de Fe.P.R.A., en donde se contempla el respeto por la intimidad y privacidad de las personas, así como su autonomía y bienestar.

A su vez, los resultados obtenidos por cada niño o niña se entregaron únicamente a familiares en sobres cerrados, manteniendo la confidencialidad de los datos. Las devoluciones se facilitaron a los responsables legales de los niños por intermedio de la institución educativa. Este requisito, ligado al principio que refiere al derecho a la privacidad de todo ser humano, favorece el resguardo de la subjetividad (Degiorgi, 2015), como así también la protección de la seguridad y la dignidad de los participantes de la muestra (Fe.P.R.A., 2013).

\section{RESULTADOS}

Se realizó un análisis estadístico teniendo en cuenta tres aspectos básicos: medidas de tendencia central, medidas de dispersión o variabilidad y distribución de frecuencias tal como se muestran a continuación. 


\section{Tabla 1}

Distribución de frecuencias de acuerdo a la edad de la muestra

\begin{tabular}{cccc}
\hline & & Frecuencia & Porcentaje \\
\hline Validos & 8 & 43 & 43,4 \\
9 & 56 & 56,6 \\
Total & 99 & 100,0 \\
\hline
\end{tabular}

Del total de 99 casos evaluados, el $43,4 \%$ de los niños y niñas tenía ocho años y el $56,6 \%$ de los niños y niñas tenían nueve años al momento de la toma.

Tabla 2

Distribución de frecuencias de acuerdo al grado en el que asistió la muestra.

\begin{tabular}{|c|c|c|}
\hline & Frecuencia & Porcentaje \\
\hline Validos 3 & 43 & 43,4 \\
\hline 4 & 56 & 56,6 \\
\hline Total & 99 & 100,0 \\
\hline
\end{tabular}

Del total de 99 casos evaluados, el $43,4 \%$ asiste al tercer grado de educación primaria y el $56,6 \%$ asiste al cuarto grado de educación primaria.

Tabla 3

Distribución de frecuencias en relación al género de la muestra.

\begin{tabular}{ccc}
\hline & Frecuencia & Porcentaje \\
\hline Validos Femenino & 55 & 55,6 \\
Masculino & 44 & 44,4 \\
Total & 99 & 100,0 \\
\hline
\end{tabular}

Del total de 99 casos evaluados, el 55,6\% pertenecen al género femenino y el 44,4\% forman parte el género masculino.

\section{Tabla 4}

Descriptivos de variables numéricas. Media, error típico de la media y desviación standard.

\begin{tabular}{crrr}
\hline & \multicolumn{1}{c}{ Media } & Erro típico & Desvi. Típica \\
\hline Validos Edad & 8,57 &, 050 &, 498 \\
IG total & 112,78 & 1,299 & 12,797 \\
IG PG & 23,06 & 2,724 & 25,552 \\
\hline
\end{tabular}


Se observa que la media en torno a la edad es de $8,57 \pm 0,050$, mientras que la desviación típica corresponde al valor de,498. En cuanto a la media en torno a la inteligencia emocional es de 112,78 $\pm 1,229$, mientras que la desviación típica corresponde al valor 12,797. Por su lado, la media en relación a la Inteligencia general obtenida de la Batería de Aptitudes Generales y diferenciales es de 23,06 $\pm 2,724$, mientras que la desviación típica es de 25,552.

\section{Tabla 5}

Distribución de frecuencia de la totalidad de casos evaluados. Casos con Altas Capacidades Intelectuales, Talento Simple y Talento compuesto.

\begin{tabular}{llcc}
\hline & & Frecuencia & Porcentaje \\
\hline Válidos & NO ACI & 46 & 46,5 \\
& ACI & 20 & 20,2 \\
& Talento Simple & 6 & 6,1 \\
& Talento Múltiple & 16 & 16,2 \\
& & \\
Incompleto & 11 & 11,1 \\
Total & 99 & 100,0 \\
\hline
\end{tabular}

Del $100 \%$ de casos evaluados, los valores reflejan que $42,5 \%$ de los niñas y niños evaluados presentan indicadores de altas capacidades intelectuales (incluye: ACl; Talento simple; Talento Múltiple) y el 46,5\% de los niñas y niños evaluados, no cuenta con las características necesarias para ser considerado dentro del rango de las Altas Capacidades Intelectuales; el 6,1\% de las niñas y niños evaluados presentan Talento Simple; el $16,2 \%$ de las niños y niñas evaluados presentan un Talento Múltiple y por último el $11 \%$ de los niñas y niños evaluados no culminó la batería.

Tabla 6

Distribución de Frecuencias Cuestionario de Inteligencia Emocional Bar-On

\begin{tabular}{ccc}
\hline & Frecuencia & Porcentaje \\
\hline Validos IE BAJA & 4 & 4,0 \\
IE PROMEDIO & 32 & 32,3 \\
IE ALTA & 61 & 61,6 \\
Incompleto & 2 & 2,2 \\
Total & 99 & 100,0 \\
\hline
\end{tabular}

De un total de 99 casos evaluados en relación a la Inteligencia emocional y a los valores esperados para la edad, el $4 \%$ obtuvo puntuaciones bajas; el $32,3 \%$ puntuaciones promedio; y por último un $61,6 \%$ ascienden a puntuaciones altas. Solo el $2 \%$ de la muestra no terminó la evaluación. 


\section{Tabla 7}

Tabla de relación de $\mathrm{ACl}$, talento simple y múltiple, en relación a la Inteligencia Emocional.

\begin{tabular}{llcccc}
\hline & & IE & IE & IE & \\
& & BAJA & PROMEDIO & ALTA & TOTAL \\
\hline Válidos & ACI & 0 & 8 & 12 & 20 \\
& Talento Simple & 1 & 2 & 3 & 6 \\
& Talento Múltiple & 0 & 2 & 14 & 16 \\
& TOTAL & 1 & 12 & 29 & 42 \\
\hline
\end{tabular}

De la totalidad de la muestra, 42 casos presentaron indicadores de Altas Capacidades Intelectuales. De ellos, 20 niños y niñas obtuvieron indicadores sin alcanzar los parámetros requeridos para ser incluidos en las categorías de "talento simple" o "múltiple", los cuales, en relación a las puntuaciones de Inteligencia Emocional, 8 obtuvieron puntuaciones dentro del valor "promedio" y 12 se destacaron con puntuaciones "altas". A la vez, se identificaron 6 niños y niñas con "Talentos Simples", de los cuales 1 obtuvo puntuaciones bajas, 2 obtuvieron puntuaciones promedio y 3 puntuaciones altas. Por último, se identificaron 16 niños y niñas con "Talentos Múltiples", de los cuales 2 ascendieron a puntuaciones promedio y el resto, 14 niños y niñas, obtuvieron puntuaciones altas en relación a la inteligencia emocional.

\section{Tabla 8}

Relación existente entre Inteligencia Emocional y niños-niñas con o sin Altas Capacidades Intelectuales.

\begin{tabular}{llccccc}
\hline & & IE & IE & IE & Bar-On & \\
& BAJA & PROMEDIO & ALTA & incompleto & TOTAL \\
\hline Válidos & ACI & 1 & 12 & 29 & $0 \%$ & 42 \\
& & $1,0 \%$ & $12,1 \%$ & $29,3 \%$ & & $42,4 \%$ \\
& NO ACI & 3 & 14 & 29 & $0 \%$ & 46 \\
& & $3 \%$ & $14,1 \%$ & $29,3 \%$ & & $46,5 \%$ \\
BADyG E2 & 0 & $6 \%$ & $6,1 \%$ & $3,0 \%$ & $2,0 \%$ & $11,1 \%$ \\
Incompleto & $0 \%$ & $42,3 \%$ & $61,6 \%$ & $2,0 \%$ & $100,0 \%$ \\
\hline
\end{tabular}

De la totalidad de la muestra, el 42,4\% presentó indicadores de alta capacidad intelectual, quienes en el test de Inteligencia Emocional obtuvieron puntuaciones "altas" (el 29,3\%), "promedio" (el 12,1\%) y "bajas" (sólo el $1,0 \%$ ). A la vez, del $46,5 \%$ que no ha presentado indicadores de altas capacidades intelectuales, se obtuvieron puntuaciones "altas" (el 29,3\%), "promedio" (el 14,1\%) y "bajas" (3,0\%) en relación a lo esperado para niños y niñas de su edad. Y finalmente, un 11,1\% de la muestra, no finalizó la Batería de Aptitudes Generales y Diferenciales E2-r, pero si realizó el Cuestionario de Inteligencia Emocional obteniendo los siguientes resultados: un 6,1\% puntuaciones "promedio"; un 3,0\% "alta". Solo dos niños de la muestra no terminaron la evaluación de Inteligencia emocional. 
Tabla 9

Prueba de Chi-Cuadrado

\begin{tabular}{|c|c|c|c|}
\hline & Valor & Gyl & $\begin{array}{ll}\begin{array}{l}\text { Sig. } \\
\text { (bilateral) }\end{array} & \text { Asintónica } \\
\end{array}$ \\
\hline $\begin{array}{l}\text { Chi-cuadrado de } \\
\text { Perason }\end{array}$ & ,974 & 2 & 614 \\
\hline $\begin{array}{l}\text { Razón de } \\
\text { verosimilitudes }\end{array}$ & 1,019 & 2 & ,601 \\
\hline $\begin{array}{l}\text { Asociación lineal } \\
\text { por lineal }\end{array}$ & ,681 & 1 & ,409 \\
\hline $\mathrm{N}$ de casos válidos & 88 & & 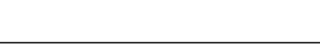 \\
\hline
\end{tabular}

De acuerdo a los resultados obtenidos, no se encontró que las variables ACl e IE estén asociadas. Es decir, no se encontró que las y los niños con ACl tengan algún nivel determinado de IE.

\section{DISCUSIÓN}

Luego de la presentación de lo anteriormente expuesto y retomando el objetivo general de la presente investigación: "Conocer la relación entre Altas Capacidades Intelectuales (ACI) e Inteligencia Emocional (IE) en niños y niñas de entre 8 y 10 años de la Ciudad de Córdoba, Argentina", se puede decir que es preciso ampliar las investigaciones acerca del constructo "Inteligencia Emocional" en relación a la alta inteligencia, ya que numerosos autores en diversas investigaciones acuerdan cierta correlación entre ésta y las Altas Capacidades Intelectuales, relación que en la presente muestra no se ha hallado.

Este trabajo se llevó a cabo en el contexto de la Práctica Supervisada en Investigación (PSI) en el Servicio de Neuropsicología Infantil (SNPI) de la Facultad de Psicología de la Universidad Nacional de Córdoba, que tiene como objeto la detección temprana Altas Capacidades Intelectuales (ACI) en la infancia.

A partir de los '90, se inician los estudios con alumnos de altas habilidades (superdotados y talentos) y de habilidades medias con el objetivo de precisar si la IE de aquéllos es diferente de la que manifiestan sus compañeros (Belmonte Lillor, 2013), motivo y objetivo que se vuelve a retomar en la presente investigación.

Si bien los principales resultados de las distintas investigaciones citadas en apartados anteriores muestran claras diferencias en el cociente emocional total que favorecen al alumnado con superdotación y talento, a partir de los resultados obtenidos en la presente investigación, se puede evidenciar que los datos no mostraron diferencias estadísticamente significativas en la percepción sobre la IE de alumnos/as que alcanzaron los niveles de indicadores $\mathrm{ACl}$ y aquellos que no, de la totalidad de la muestra estudiada, tal es así que, a diferencia de Schewean et al. (2006), entre la autopercepción del alumnado no superdotado o talentoso y la autopercepción de quienes presentaron indicadores de $\mathrm{ACl}$ no se encontraron diferencias significativas.

En general, los alumnos/as con superdotación y talento del presente estudio autoinformaron un buen ajuste emocional, buena capacidad de adaptabilidad y habilidades intra e interpersonales, así como adecuadas estrategias para manejar el estrés. Los datos recabados, entonces, no se muestran acordes con algunos autores que mantienen la idea de que los alumnos con superdotación tienen más riesgo de presentar problemas con sus iguales (Fieldler, 1998; Morelock, 1992; Silverman, 1993).

Sin embargo, nuestros resultados coinciden con los hallados por autores que sugieren que los alumnos con superdotación y talento no presentan problemas socioemocionales (Bellamy, Gore, y Sturgis, 2005; Ferrando, Ferrándiz, Prieto, Sánchez, Hernández, Serna y López, 2007; Schewean, Saklofske, Widdifield-Konkin, Parker y Kloosterman, 2006) en relación a otros niños de su misma edad sin talento o superdotación.

Es preciso señalar que no hemos encontrado estudios en los que se profundice en la Inteligencia Emocional del superdotado y el talento en las edades de la Educación Primaria, a excepción, del realizado por Sánchez 
(2006), quien no halló diferencias estadísticamente significativas utilizando un cuestionario de Inteligencia Emocional autopercibida, en el que se valoraban las habilidades de autoconciencia, autocontrol, aprovechamiento emocional, habilidad social y empatía. Consideramos, por ello, necesario la realización de más estudios que permitan contrastar los resultados aquí obtenidos.

Además, si bien Hollingworth (1942) y diversos autores consideran que los niños y niñas con una inteligencia muy elevada son propensos a los problemas de ajuste social y emocional, aquí se observa que el grupo con talento mantienen puntuaciones elevadas alcanzando un cociente emocional alto, pudiéndose observar entonces lo planteado por Freeman (1983) quién no encontró diferencias en las puntuaciones de desviación emocional cuando comparó alumnos de alta habilidad con un grupo control.

Entonces, quizás, los niños y niñas superdotados/as y talentosos/as tienen las mismas necesidades emocionales que otros niños/as y las mismas necesidades educativas de expresión y exploración, pero la diferencia reside en su intensidad (Freeman, 1979), tal como sostiene Sword (2005) cuando afirma que la complejidad cognitiva de estos alumnos va de la mano de la complejidad emocional, siendo sus vivencias emocionales más complejas e intensas que las de sus compañeros/as.

Así, tal como se evidencia desde el grupo de investigadores (Baer, 1991; Freeman, 1983), los niños y niñas superdotadas no tienen mayores problemas emocionales ni sociales que sus compañeros no superdotados. En general, los detractores de este punto de vista creen que los alumnos/as superdotados son capaces de comprenderse mejor a sí mismos y a los otros debido a sus acentuadas capacidades intelectuales, y por tanto lidiar mejor con el estrés, el conflicto y la asincronía en el desarrollo (Garland \& Zigler, 1999).

Finalmente, si bien Roedell (1986) sostiene que, a mayor grado de superdotación, mayor es la probabilidad de que el alumno experimente problemas de ajuste, Grossberg \& Cornell (1988) consideran que no es la habilidad en sí misma la responsable y que los niños superdotados experimentan problemas de ajuste por las mismas razones que otros niños: por temas relacionados con la familia o la escuela.

La investigación en este campo muestra que el concepto de IE ha adquirido una base sólida científica y que comienza a madurar como marco de estudio. Es así que siguiendo a Cobb y Mayer (2000) "Los próximos años seguramente nos depararán interesantes hallazgos en el ámbito educativo que pondrán aún más de

24 relieve el papel potencial de la IE en las aulas y la necesidad de integrar en el currículo el desarrollo de las habilidades de IE".

Es importante incluir en los procesos de identificación herramientas que nos permitan estudiar la configuración emocional de los alumnos con alta habilidad (superdotados y talentos) porque éstos, al igual que sus compañeros, aprenden mejor en ambientes donde se favorecen las emociones, se fomenta la motivación y se alienta la implicación en la tarea.

\section{CONCLUSIONES}

De un total de 99 casos a los que se les administró la Batería de Aptitudes Generales y Diferenciales BADyG E2-r no se detectó superdotación, no obstante, se identificó que el 42,5\% del total la muestra cuenta con indicadores $\mathrm{ACl}$; de ese $42,5 \%$ se desprende que el $6,1 \%$ de los niños y niñas presentaron talento simple y $16,2 \%$ de los niños y niñas presentaron talento complejo. De estos resultados se desprende la importancia de la detección temprana, necesaria para poner en marcha distintas estrategias atinentes a desarrollar de manera integral las potencialidades de los niños.

De la totalidad de la muestra a los que se les administró el Cuestionario de Inteligencia General Bar-On, se encontraron los siguientes resultados: $32 / 99$ casos con inteligencia emocional promedio para los niños y niñas de su edad; 61/99 casos con inteligencia emocional alta para los niños y niñas de su edad; 4/99 casos que obtuvieron puntuaciones bajas en relación a lo esperado para niños y niñas de su edad y 2/99 casos los cuales no completaron la batería. De acuerdo a los datos presentamos observamos que la Inteligencia Emocional de alto nivel está presente en un $61 \%$ de la totalidad de la muestra.

Cuando se correlacionaron los datos, se obtuvieron resultados sorprendentes, ya que se partió de una hipótesis inicial en la que los niños con altas capacidades intelectuales tuvieran una alta inteligencia emocional; pero esto no fue así, ya que hubo una gran variabilidad de resultados, dándole un lugar de relevancia a la inteligencia emocional, sin que la misma tenga que aparecer sólo cuando las Altas Capacidades se hacen presentes. Finalmente, a raíz de los datos obtenidos, se podrían generar nuevas estrategias y herramientas 
que aborden el constructo de Inteligencia Emocional en la Infancia, con el objetivo de fomentar el máximo desarrollo de la misma en edades tempranas

\section{REFERENCIAS}

Baer, J. (1991). "Depression, general anxiety, test anxiety, and rigidity of gifted junior high and high school children”. Psychological Reports, 69, 1128-1130.

Bar-On, R. (1997). The Bar-On Emotional Quotient Inventory (EQ-i): A Test of Emotional Intelligence. Toronto, Canadá: Multi-Health Systems.

Belmonte Lillo V. M (2013). Inteligencia Emocional y Creatividad: Factores Predictores del Rendimiento. Trabajo de Tesis doctoral. Universidad de Murcia, Facultad de Educación, Departamento de Psicología Evolutiva y de la Educación.

Bellamy, A., Gore, D. \& Sturgis, J. (2005). “Una exploración de la relevancia de la inteligencia emocional en los programas educativos para los estudiantes de alta capacidad". Electronic Journal of Research in Educational Psychology, 6-3 (2), 53-78.

Bologna, E. (2011) Técnicas de muestreo. Estadística para psicología y educación. Editorial Brujas: Córdoba.

Castelló, A. (2008). Bases intelectuales de la excepcionalidad: un esquema integrador. Revista Española de Pedagogía, 66(240), 203-220.

Coob, C. \& Mayer, J.D. (2000). “Emotional intelligence: What the research says”. Educational Leadership, 58, 14-18.

Código de Ética de la Federación de Psicólogos de la República Argentina (FePRA) (2013). Cap. 2. Normas Deontológicas. Art. 1: Consentimiento Informado, Art. 2: Secreto Profesional y Art. 4: Investigación. Argentina, 10 de abril del año 1999. Modificado por Asamblea Extraordinaria el 30 de noviembre del año 2013.

Código de Ética de la Provincia de Córdoba (1987). Cap. VI: De la Investigación y Docencia. Art. 18. Consejo de Psicólogos de la Provincia de Córdoba. Resolución N5. Córdoba, Argentina, 19 de septiembre del año 1987.

Comes, G. Díaz, E; Luque, A. \& Moliner, O (2008) La evaluación psicopedagógica del alumnado con altas capacidades intelectuales. Educación Inclusiva, ISSN-e 1889-4208, 1 (1).

Degiorgi, G. M. (2015) La dimensión Ética y Deontológica en el ejercicio profesional del psicólogo. Ficha de cátedra de Deontología y Legislación Profesional.

Ferrándiz García, C. (2011). Estrategias Metodológicas de Aprendizaje Activo para el Alumnado con Altas Habilidades. Documento: Documento general proceso de identificación del alumnado con alta habilidad intelectual. Dpto. Psicología Evolutiva y de la Educación. Grupo Investigación Altas Habilidades. Universidad de Murcia, España.

Ferrando, M., Ferrándiz, C., Prieto, L., Sánchez, C., Hernández, D., Serna, B. \& López, J. A. (2007). En: Patti, Jannet; Brackett, Marc; Ferrándiz, Carmen \& Ferrando, Mercedes (2011). ¿Por qué y cómo mejorar la inteligencia emocional de los alumnos superdotados? REIFOP, 14 (3).

Ferrando, M. (2006). Creatividad e inteligencia emocional: un estudio empírico en alumnos con altas habilidades. Published PhD Thesis. Universidad de Murcia.

Fieldler, E.D. (1998). "Denial of anger/denial of self Dealing with the dilemas". Roeper Review, 20 (3), 158-161.

Freeman, J. (1979). Gifted Children. Lancaster, UK: MTP Press.

Freeman, J. (1983). "Emotional Problems of the Gifted Child". Journal of Child Psychology and Psychiatry, 24. (3), $481-485$.

Gardner, H. (1983). Frames of mind: The theory of multiples intelligences. New York: Basic.

Garland, A. F. \& Zigler, E. (1999). "Emotional and behavioural problems among highly intellectually gifted youth". Roeper Review, 22 (1), 41-44.

Goleman, D. (1995). Inteligencia Emocional. Barcelona: Ed. Kairós.

Grossberg, L. N. \& Cornell, D. G. (1988). “Relationship between personality adjustment and high intelligence: Terman versus Hollingworth". Exceptional Children, 55, 266-272.

Hernández Sampieri, R., Fernández-Collado C., y Baptista Lucio P. (2006). Metodología de la Investigación. México: McGraw-Hill Interamericana Editores.

Hollingworth, L. (1942). Children above 180 IQ Stanford Binet. New York: World Book. 
Irueste, P. \&Ferrándiz, C. (2016) Validación de la Batería de Aptitudes Diferenciales y Generales (BADyG) en niños de 4 a 6 años de Córdoba, Argentina. I Congreso Internacional de Nuevas Perspectivas en el Estudio de la Superdotación y el Talento. Murcia, España.

Ley de Educación Provincial No 9870 (2010). Cap. 1. Sección Segunda. Art. 22. Inc. "a"y “d"; Cap. 2. Sección Segunda. Art. 12. Inc. "a" y"b"; y Cap. 3. Sección Segunda. Art. 49. Dirección General de Educación Superior, Ministerio de Educación de Córdoba. Córdoba, Argentina, 15 de Diciembre del año 2010.

Manzano, A. \& Arranz, E. B. (2008) Contexto familiar, superdotación, talento y altas capacidades. Anuario de Psicología, 39 (3), 289-309. Recuperado en: http://www.redalyc.org/articulo.oa?id=97021031001.

Mayer, J. D. \& Salovey, P. (1997). What is emotional intelligence? In P. Salovey \& D. Sluyter (Eds). Emotional Development and Emotional Intelligence: Implications for Educators (p. 3-31) Nueva York: Basic Books.

Montero, I. \& León, O. G. (2007) Guía para nombrar los estudios de investigación en Psicología. International Journal of Clinical and Health Psychology, 7 (3), 847-862.

Morelock, M.J. (1992). "Giftedness: The view from within". Understanding Our Gifted, 4 (3), 11-15.

Patti, Jannet; Brackett, Marc; Ferrándiz, Carmen \& Ferrando, Mercedes (2011). ¿Por qué y cómo mejorar la inteligencia emocional de los alumnos superdotados? REIFOP, 14 (3).

Prieto, M. D., Ferrándiz, C., Ferrando, M., Sánchez, C., \& Bermejo, R. (2008). Inteligencia emocional y alta habilidad.

Roedell, W.C. (1986). "Socioemotional vulnerabilities of young gifted children". Journal of Children in Contemporary Society, 18, (3-4), 17-29.

Sainz, Marta; Soto, Gloria; Almeida, Leandro; Ferrándiz, Carmen; Fernández, Ma Carmen \& Ferrando, Mercedes (2011). Competencias socio-emocionales y creatividad según el nivel de inteligencia. REIFOP, 14 (3).

Sastre-Riba, S. (2008). Niños con altas capacidades y su funcionamiento cognitivo diferencial (Síntesis). Rev Neurol, 46(Supl 1), S11-S16.

Schwean, V. L., Saklofske, D.H., Widdified-Konkin, L., Parker, J. \& Kloosterman, P. (2006). “Emotional Intelligence and Gifted Children". E-Journal of Applied Psychology: Emotional Intelligence, 2 (2), 30-37.

Silverman, L. K. (1993). Counseling the Gifted and Talented. Denver, CO: Love.

26 Sword, L. K. (2005). Emotional intensity in gifted children. Recuperado de: www.sengifted.org (14-07-2011).

Yuste Hernanz, C. \& Yuste Peña, D. (2014) Batería de Aptitudes Diferenciales y Generales. Preguntas frecuentes. Manual web. Madrid, España: CEPE. Recuperado enhttps://sites.google.com/site/gestorbadyg/manual-web-indice.

Fecha de Recepción: 29/06/2019

Fecha de Aceptación: 22/10/2019 